\title{
INTERLACED DENSE POINT AND ABSOLUTELY CONTINUOUS SPECTRA FOR HAMILTONIANS WITH CONCENTRIC-SHELL SINGULAR INTERACTIONS
}

\author{
Pavel Exner and Martin Fraas \\ Nuclear Physics Institute, Czech Academy of Sciences, 25068 Řež near Prague, \\ Doppler Institute, Czech Technical University, Břehová 7, 11519 Prague, Czechia \\ e-mail: exner@ujf.cas.cz,fraas@ujf.cas.cz
}

\begin{abstract}
We analyze the spectrum of the generalized Schrödinger operator in $L^{2}\left(\mathbb{R}^{\nu}\right), \nu \geq 2$, with a general local, rotationally invariant singular interaction supported by an infinite family of concentric, equidistantly spaced spheres. It is shown that the essential spectrum consists of interlaced segments of the dense point and absolutely continuous character, and that the relation of their lengths at high energies depends on the choice of the interaction parameters; generically the p.p. component is asymptotically dominant. We also show that for $\nu=2$ there is an infinite family of eigenvalues below the lowest band.
\end{abstract}

PACS number: 03.65.Xp keywords: Schrödinger operators, singular interactions, absolutely continuous spectrum, dense pure point spectrum

\section{Introduction}

Quantum systems with the spectrum consisting of components of a different nature attract attention from different points of view. Probably the most important among them concerns random potentials in higher dimensions a demonstration of existence of a mobility edge is one of the hardest questions of the present mathematical physics. At the same time, a study of specific non-random systems can reveal various types of spectral behaviour which differ from the generic type.

An interesting example among these refers to the situation where the spectrum is composed of interlacing intervals of the dense point and absolutely continuous character. A way to construct such models using radially 
periodic potentials was proposed in [1]: since at large distances in such a system the radial and angular variables "almost decompose" locally and the radial part behaves thus essentially as one-dimensional there are spectral intervals where the particle can propagate, with the gaps between them filled densely by localized states.

To be specific consider, e.g., the operator $\mathrm{t}=-\mathrm{d}^{2} / \mathrm{d} x^{2}+q(x)$ on $L^{2}(\mathbb{R})$ with $q$ bounded and periodic. By the standard Floquet analysis the spectrum of $\mathrm{t}$ is purely absolutely continuous consisting of a family of bands, $\sigma(\mathrm{t})=\bigcup_{k=0}^{N}\left[E_{2 k}, E_{2 k+1}\right]$, corresponding to a strictly increasing, generically infinite sequence $\left\{E_{k}\right\}_{k=0}^{N}$. Suppose now that the potential is mirror-symmetric, $q(x)=q(-x)$, and consider the operator

$$
\mathrm{\top}=-\triangle+q(|x|)
$$

on $L^{2}\left(\mathbb{R}^{\nu}\right), \nu \geq 2$. It was shown in [1] that the essential spectrum of $T$ covers the half-line $\left[E_{0}, \infty\right)$, being absolutely continuous in the spectral bands of $\mathrm{t}$ and dense pure point in the gaps $\left(E_{2 k-1}, E_{2 k}\right), k=1, \ldots N$.

The well-known properties of one-dimensional Schrödinger operators tell us that the dense point segments in this example shrink with increasing energy at a rate determined by the regularity of the potential. If we replace the bounded $q$ by a family of $\delta$ interactions, the segment lengths tend instead to a positive constant [2], nevertheless, the absolutely continuous component still dominates the spectrum at high energies.

The aim of this paper is to investigate a similar model in which a family of concentric, equally spaced spheres supports generalized point interactions with identical parameters. We will demonstrate that the interlaced spectral character persists and, depending on the choice of the parameters, each of the components may dominate in the high-energy limit, or neither of them. Specifically, the ratio of the adjacent $p p$ and $a c$ spectral segments, $\left(E_{2 k}-\right.$ $\left.E_{2 k-1}\right) /\left(E_{2 k+1}-E_{2 k}\right)$, has three possible types of behaviour, namely like $\mathcal{O}\left(k^{\mu}\right)$ with $\mu=0, \pm 1$. What is more, in the generic case we have $\mu=1$ so the dense point part dominates, which is a picture very different from the mobility-edge situation mentioned in the opening. Apart of this main result, we are going to show that the interesting result about existence of the so-called "Welsh eigenvalues" in the two-dimensional case[3, 4] also extends to the case of generalized point interactions. 


\section{The model}

As we have said, we are going to investigate generalized Schrödinger operators in $\mathbb{R}^{\nu}, \nu \geq 2$, with spherically symmetric singular interaction on concentric spheres, the radii of which are supposed to be $R_{n}=n d+d / 2, n \in \mathbb{N}$. It is important that the system is radially periodic, hence the interactions on all the spheres are assumed to be the same. In view of the spherical symmetry we may employ the partial-wave decomposition: the isometry $\mathrm{U}$ : $L^{2}\left((0, \infty), r^{\nu-1} d r\right) \rightarrow L^{2}(0, \infty)$ defined by $\cup f(r)=r^{\frac{\nu-1}{2}} f(r)$ allows us to write $L^{2}\left(R^{\nu}\right)=\bigoplus_{l \in \mathbb{N}_{0}} \mathrm{U}^{-1} L^{2}(0, \infty) \otimes S_{l}$, where $S_{l}$ is the $l$-th eigenspace of the Laplace-Bertrami operator on the unit sphere. The operator we are interested in can be then written as

$$
\mathrm{H}_{\Lambda}:=\bigoplus_{l} \mathrm{U}^{-1} \mathrm{H}_{\Lambda, l} \mathrm{U} \otimes \mathrm{I}_{l}
$$

where $\mathrm{I}_{l}$ is the identity operator on $S_{l}$ and the $l$-th partial wave operators

$$
\mathrm{H}_{\Lambda, l}:=-\frac{d^{2}}{d r^{2}}+\frac{1}{r^{2}}\left[\frac{(\nu-1)(\nu-3)}{4}+l(l+\nu-2)\right]
$$

are determined by the boundary conditions ${ }^{1}$ at the singular points $R_{n}$,

$$
\left(\begin{array}{c}
f\left(R_{n}+\right) \\
f^{\prime}\left(R_{n}+\right)
\end{array}\right)=e^{i \chi}\left(\begin{array}{cc}
\gamma & \beta \\
\alpha & \delta
\end{array}\right)\left(\begin{array}{c}
f\left(R_{n}-\right) \\
f^{\prime}\left(R_{n}-\right)
\end{array}\right) ;
$$

in the transfer matrix $\Lambda:=e^{i \chi}\left(\begin{array}{c}\gamma \beta \\ \alpha \delta\end{array}\right)$ the parameters $\alpha, \beta, \gamma, \delta$ are real and satisfy the condition $\alpha \beta-\gamma \delta=-1$. In other words, the domain of the selfadjoint operator $\mathrm{H}_{\Lambda, l}$ is

$$
\begin{gathered}
D\left(\mathrm{H}_{\Lambda, l}\right)=\left\{f \in L^{2}(0, \infty): f, f^{\prime} \in A C_{\mathrm{loc}}\left((0, \infty) \backslash \cup_{n}\left\{R_{n}\right\}\right) ;\right. \\
-f^{\prime \prime}+\frac{1}{r^{2}}\left[\frac{(\nu-1)(\nu-3)}{4}+l(l+\nu-2)\right] f \in L^{2}(0, \infty) ; \\
\left.F\left(R_{n}+\right)=\Lambda F\left(R_{n}-\right)\right\},
\end{gathered}
$$

where the last equation is a shorthand for the boundary conditions (2.3). If the dimension $\nu \leq 3$ we have to add a condition for behaviour of $f \in D\left(\mathrm{H}_{l}\right)$ at the origin: for $\nu=2, l=0$ we assume that $\lim _{r \rightarrow 0+}[\sqrt{r} \ln r]^{-1} f(r)=0$, and for $\nu=3, l=0$ we replace it by $f(0+)=0$. Since the generalized point interaction is kept fixed, we will mostly drop the symbol $\Lambda$ in the following.

\footnotetext{
${ }^{1}$ For relations of these conditions to the other standard parametrization of the generalized point interaction, $(U-I) F\left(R_{n}\right)+i(U+I) F^{\prime}\left(R_{n}\right)=0$, see [?]
} 


\section{Generalized Kronig-Penney model}

As in the regular case the structure of the spectrum is determined by the underlying one-dimensional Kronig-Penney model. We need its generalized form where the Hamiltonian acts as the one dimensional Laplacian except at the interaction sites, $x_{n}:=n d+d / 2, n \in \mathbb{Z}$, where the wave functions satisfy boundary conditions analogous to (2.3). To be explicit we consider the four-parameter family of self-adjoint operators

$$
\mathrm{h}_{\Lambda} f:=-f^{\prime \prime}, D\left(\mathrm{~h}_{\Lambda}\right)=\left\{f \in \mathcal{H}^{2,2}\left(\mathbb{R} \backslash \cup_{n}\left\{x_{n}\right\}\right): F\left(x_{n}+\right)=\Lambda F\left(x_{n}-\right)\right\}
$$

with the matrix $\Lambda$ same as above (without loss of generality we may assume $\chi=0$ because it is easy to see that operators differing by the value of $\chi$ are isospectral). Spectral properties of this model were investigated in $[6,7]$ where it was shown that the following three possibilities occur:

(i) the $\delta$-type: $\beta=0$ and $\gamma=\delta=1$. In this case the gap width is asymptotically constant; it behaves like $2|\alpha| d^{-1}+\mathcal{O}\left(n^{-1}\right)$ as the band index $n \rightarrow \infty$. This is the standard Kronig-Penney model.

(ii) the intermediate type: $\beta=0$ and $|\gamma+\delta|>2$. Now the quotient of the band width to the adjacent gap width is asymptotically constant behaving as $\arcsin \left(2|\delta+\gamma|^{-1}\right) / \arccos \left(2|\delta+\gamma|^{-1}\right)+\mathcal{O}\left(n^{-1}\right)$.

(iii) the $\delta^{\prime}$-type: the generic case, $\beta \neq 0$. In this case the band width is asymptotically constant; it behaves like $8|\beta d|^{-1}+\mathcal{O}\left(n^{-1}\right)$ as $n \rightarrow \infty$.

Recall that these types of spectral behaviour correspond to high-energy properties of a single generalized point interaction as manifested through scattering, resonances [5], etc.

There is one more difference from standard Floquet theory which we want to emphasize. It is well known [8] that in the regular case the spectral edge $E_{0}$ corresponds to a symmetric eigenfunction. In the singular case this is no longer true; one can check easily the following claim.

Proposition 3.1 Let $u$ be an $\alpha$-periodic solution of the equation $-u^{\prime \prime}=E_{0} u$ on $(-d / 2, d / 2)$ with $U\left(x_{n}+\right)=\Lambda U\left(x_{n}-\right)$, where $E_{0}:=\inf \sigma\left(\mathrm{h}_{\Lambda}\right)$. Then $u$ is periodic for $\beta \geq 0$ and antiperiodic for $\beta<0$.

To finish the discussion of the one-dimensional comparison operator, let us state three auxiliary results which will be needed in the next section. 
Lemma 3.2 There is a constant $C>0$ such that for every function $u$ in the domain of the operator $\mathrm{h}_{\Lambda}$ it holds that

$$
\left\|u^{\prime}\right\| \leq C\left(\left\|\mathrm{~h}_{\Lambda} u\right\|+\|u\|\right) .
$$

Proof: We employ Redheffer's inequality [9] which states that

$$
\int_{a}^{b}\left|u^{\prime}(x)\right|^{2} \mathrm{~d} x \leq C^{\prime}\left(\int_{a}^{b}\left|u^{\prime \prime}(x)\right|^{2} \mathrm{~d} x+\int_{a}^{b}|u(x)|^{2} \mathrm{~d} x\right)
$$

holds for any $u$ twice differentiable in an interval $[a, b]$ and some $C^{\prime}>0$; then we get an inequality similar to (3.2) for the squares of the norms by summing up these inequalities with $a=x_{n}, b=x_{n+1}$, and the sought result with $C=2 C^{\prime}$ follows easily.

Lemma 3.3 The set of functions from $D\left(\mathrm{~h}_{\Lambda}\right)$ with a compact support is a core of the operator $\mathrm{h}_{\Lambda}$.

Proof: To a given $u \in D\left(\mathrm{~h}_{\Lambda}\right)$ and $\varepsilon>0$ we will construct an approximation function $u_{\varepsilon} \in D\left(\mathrm{~h}_{\Lambda}\right)$ which is compactly supported to the right, i.e. it satisfies $\sup \operatorname{supp} u_{\varepsilon}<\infty$, and

$$
\int_{\mathbb{R}}\left(\left|u-u_{\varepsilon}\right|^{2}+\left|u^{\prime \prime}-u_{\varepsilon}^{\prime \prime}\right|^{2}\right)(t) \mathrm{d} t \leq \varepsilon .
$$

Given $x \in \mathbb{R}$ and $d>0$ we can employ for a function $v \in \mathcal{H}^{2,2}(x, x+d)$ the Sobolev embedding,

$$
|v(x)|^{2}+\left|v^{\prime}(x)\right|^{2} \leq \sup _{t \in[x, x+d]}|v(t)|^{2}+\left|v^{\prime}(t)\right|^{2} \leq C_{1} \int_{x}^{x+d}\left(|v|^{2}+\left|v^{\prime \prime}\right|^{2}\right)(t) \mathrm{d} t
$$

with a constant $C_{1}$ which depends on $d$ but not on $x$. Let us take next a pair of functions, $\phi_{i} \in C^{\infty}(0, d), i=1,2$, such that they satisfy $\phi_{1}(0)=\phi_{2}^{\prime}(0)=1$ and $\phi_{1}^{\prime}(0)=\phi_{2}(0)=\phi_{i}(d)=\phi_{i}^{\prime}(d)=0$. Denote by $M_{i}$ the maximum of $\left|\phi_{i}(t)\right|^{2}+\left|\phi_{i}^{\prime \prime}(t)\right|^{2}$ and put $M:=\max \left\{M_{1}, M_{2}\right\}$; then it holds

$$
\int_{0}^{d}\left(\left|a \phi_{1}+b \phi_{2}\right|^{2}+\left|a \phi_{1}^{\prime \prime}+b \phi_{2}^{\prime \prime}\right|^{2}\right)(t) \mathrm{d} t \leq 2 M d\left(a^{2}+b^{2}\right) .
$$


In view of the assumption made about the function $u$ we can find $n$ such that $\int_{x_{n}}^{\infty}\left(|u|^{2}+\left|u^{\prime \prime}\right|^{2}\right)(t) \mathrm{d} t \leq \tilde{\varepsilon}:=\varepsilon /\left(2+8 M d C_{1}\right)$ and define

$$
u_{\varepsilon}(x):=\left\{\begin{array}{llr}
u(x) & \text { if } & x \leq x_{n} \\
u\left(x_{n}+\right) \phi_{1}(x)+u^{\prime}\left(x_{n}+\right) \phi_{2}(x) & \text { if } & x \in\left(x_{n}, x_{n}+d\right) \\
0 & \text { if } & x \geq x_{n}+d
\end{array}\right.
$$

Then $u_{\varepsilon}$ belongs to $D\left(\mathrm{~h}_{\Lambda}\right)$ being compactly supported to the right and

$$
\begin{aligned}
\int_{\mathbb{R}}\left(\left|u-u_{\varepsilon}\right|^{2}+\left|u^{\prime \prime}-u_{\varepsilon}^{\prime \prime}\right|^{2}\right)(t) \mathrm{d} t \leq 2 \int_{x_{n}}^{\infty}\left(|u|^{2}+\left|u^{\prime \prime}\right|^{2}+\left|u_{\varepsilon}\right|^{2}+\left|u_{\varepsilon}^{\prime \prime}\right|^{2}\right)(t) \mathrm{d} t \\
\leq 2 \int_{x_{n}}^{\infty}\left(|u|^{2}+\left|u^{\prime \prime}\right|^{2}\right)(t) \mathrm{d} t+8 M d\left(\left|u\left(x_{n}\right)\right|^{2}+\left|u^{\prime}\left(x_{n}\right)\right|^{2}\right) \\
\leq\left(2+8 M d C_{1}\right) \int_{x_{n}}^{\infty}\left(|u|^{2}+\left|u^{\prime \prime}\right|^{2}\right)(t) \mathrm{d} t \leq\left(2+8 M d C_{1}\right) \tilde{\varepsilon}=\varepsilon .
\end{aligned}
$$

Furthermore, one can take this function $u_{\varepsilon}$ and perform on it the analogous construction to get the support compact on the left, arriving in this way at a compactly supported $\tilde{u}_{\varepsilon}$ such that

$$
\int_{\mathbb{R}}\left(\left|u-\tilde{u}_{\varepsilon}\right|^{2}+\left|u^{\prime \prime}-\tilde{u}_{\varepsilon}^{\prime \prime}\right|^{2}\right)(t) \mathrm{d} t \leq 2 \varepsilon,
$$

and since $\varepsilon$ was arbitrary by assumption the lemma is proved.

The last one is a simple observation, which is however the main tool for conversion of the proofs in the regular case to their singular counterparts.

Lemma 3.4 Let $u, v \in D\left(\mathrm{~h}_{\Lambda}\right)$, then the Wronskian

$$
W[\bar{u}, v](x):=\bar{u}(x) v^{\prime}(x)-\bar{u}^{\prime}(x) v(x)
$$

is a continuous function of $x$ on the whole real axis.

Proof: The condition $\alpha \beta-\gamma \delta=-1$ for the transfer matrix $\Lambda$ is equivalent to $\Lambda^{*} \sigma_{2} \Lambda=\sigma_{2}$, where $\sigma_{2}$ is the second Pauli matrix[6]. Then we have

$$
\begin{gathered}
W[\bar{u}, v]\left(x_{n}+\right)=i U^{*}\left(x_{n}+\right) \sigma_{2} V\left(x_{n}+\right)=i\left(\Lambda U\left(x_{n}-\right)\right)^{*} \sigma_{2} \Lambda V\left(x_{n}-\right) \\
=i U^{*}\left(x_{n}-\right) \sigma_{2} V\left(x_{n}-\right)=W[\bar{u}, v]\left(x_{n}-\right)
\end{gathered}
$$


which concludes the proof.

The way in which we are going to employ this result is the following. Suppose we have real-valued functions $u_{0}, v_{0}, u$ which are $\mathcal{H}^{2,2}$ away from the points $x_{n}$ and satisfy the boundary conditions (2.3) at them. Let, in addition, $W\left[u_{0}, v_{0}\right]$ be nonzero - in the applications below this will be true as $u_{0}, v_{0}$ will be linearly independent generalized eigenfunctions of $h_{\Lambda}$ - then by the lemma the vector function

$$
y=\left[\begin{array}{cc}
u_{0} & v_{0} \\
u_{0}^{\prime} & v_{0}^{\prime}
\end{array}\right]^{-1}\left(\begin{array}{c}
u \\
u^{\prime}
\end{array}\right)=W\left[u_{0}, v_{0}\right]^{-1}\left(\begin{array}{c}
v_{0}^{\prime} u-v_{0} u^{\prime} \\
-u_{0}^{\prime} u+u_{0} u^{\prime}
\end{array}\right)
$$

is continuous everywhere including the points $x_{n}$.

\section{The essential spectrum}

Now we are going to demonstrate the spectral properties of $\mathrm{H}_{\Lambda}$ announced in the introduction. We follow the ideology used in the regular case [10, 1], localizing first the essential spectrum and finding afterwards the subsets where it is absolutely continuous. In view of the partial wave decomposition (2.1) it is natural to start with the partial wave operators $\mathrm{H}_{l}$.

The essential spectrum is stable under a rank one perturbation, hence adding the Dirichlet boundary condition at a point $a>0$ to each of the operators $\mathrm{H}_{l}$, $\mathrm{h}_{\Lambda}$ we do not change their essential spectrum. Moreover, multiplication by $C x^{-2}$ is a relatively compact operator on $L^{2}(a, \infty)$, thus the essential spectra of the said operators coincide,

$$
\sigma_{e s s}\left(\mathrm{H}_{l}\right)=\sigma_{e s s}\left(\mathrm{~h}_{\Lambda}\right) \text {. }
$$

With this prerequisite we can pass to our first main result.

Theorem 4.1 The essential spectrum of the operator (2.1) is equal to

$$
\sigma_{e s s}\left(\mathbf{H}_{\Lambda}\right)=\left[\inf \sigma_{e s s}\left(\mathrm{~h}_{\Lambda}\right), \infty\right) \text {. }
$$

The idea of the proof is the same as in [10]: first we check that inf $\sigma_{e s s}\left(\mathrm{H}_{\Lambda}\right)$ cannot be smaller then inf $\sigma_{\text {ess }}\left(\mathrm{h}_{\Lambda}\right)$, after that we show that $\sigma_{\text {ess }}\left(\mathrm{H}_{\Lambda}\right)$ contains the whole interval [inf $\left.\sigma_{e s s}\left(\mathrm{~h}_{\Lambda}\right), \infty\right)$. 
Proposition 4.2 Under the assumptions stated we have

$$
\inf \sigma_{e s s}\left(\mathrm{H}_{\Lambda}\right) \geq \inf \sigma_{e s s}\left(\mathrm{~h}_{\Lambda}\right) \text {. }
$$

Proof: If $\nu>2$ we infer from equations (4.1), (4.4) that

$$
\inf \sigma_{e s s}\left(\mathrm{H}_{\Lambda}\right) \geq \inf _{l} \inf \sigma\left(\mathrm{H}_{l}\right)=\inf \sigma_{e s s}\left(\mathrm{~h}_{\Lambda}\right) ;
$$

notice that with the exception of the case $\nu=2, l=0$ the centrifugal term in the partial waves operators (2.2) is strictly positive, and consequently, the mini-max principle implies

$$
\inf \sigma\left(\mathrm{H}_{l}\right) \geq \inf \sigma\left(\mathrm{h}_{\Lambda}\right)=\inf \sigma_{e s s}\left(\mathbf{H}_{l}\right) \geq \inf \sigma\left(\mathbf{H}_{l}\right) .
$$

For $\nu=2$ the argument works again, we have just to be a little more cautious and consider in the first partial wave the infimum over the essential spectrum only.

Proposition $4.3 \quad \sigma_{e s s}\left(\mathrm{H}_{\Lambda}\right) \supset\left[\inf \sigma_{e s s}\left(\mathrm{~h}_{\Lambda}\right), \infty\right]$.

Proof: The idea is to employ Weyl criterion [11]. Let $\lambda_{0} \in \sigma_{\text {ess }}\left(h_{\Lambda}\right)$ and $\lambda>0$, then we have to show that for every $\varepsilon>0$ there exists a function

$$
\phi \in D\left(\mathrm{H}_{\Lambda}\right) \quad \text { satisfying } \quad\left\|\left(\mathrm{H}_{\Lambda}-\lambda_{0}-\lambda\right) \phi\right\| \leq \varepsilon\|\phi\| .
$$

Basic properties of the essential spectrum together with Lemma 3.3 provide us with a compactly supported $u \in D\left(\mathrm{~h}_{\Lambda}\right)$ such that $\left\|u^{\prime \prime}-\lambda_{0} u\right\| \leq \frac{1}{2} \varepsilon$. in view of the periodicity we may suppose that $\operatorname{supp} u \subset(0, L)$. Next we are going to estimate $\lambda$ by the repulsive centrifugal potential in a suitably chosen partial wave. Putting $l_{R}:=[\sqrt{\lambda R}]$ we have

$$
\frac{1}{r^{2}}\left[\frac{(\nu-1)(\nu-3)}{4}+l_{R}\left(l_{R}+\nu-2\right)\right]=\lambda+\mathcal{O}\left(R^{-1}\right) \quad \text { for } \quad r \in[R, R+L]
$$

as $R \rightarrow \infty$, hence choosing $R$ large enough one can achieve that

$$
\sup _{r \in[R, R+L]}\left|\frac{1}{r^{2}}\left[\frac{(\nu-1)(\nu-3)}{4}+l_{R}\left(l_{R}+\nu-2\right)\right]-\lambda\right| \leq \frac{1}{2} \varepsilon .
$$


Next we employ the partial wave decomposition, considering a unit vector $Y \in S_{l_{n_{\varepsilon}}}$ and putting $\phi(x):=\mathrm{U}^{-1} u(|x|-R) Y(x /|x|)$. It holds obviously $\phi \in D\left(\mathrm{H}_{\Lambda}\right),\|\phi\|=\|u(\cdot-R)\|$, and $^{2}$

$$
\begin{aligned}
& \left\|\mathrm{H}_{\Lambda} \phi-\left(\lambda_{0}+\lambda\right) \phi\right\|=\left\|\mathrm{H}_{l_{R}} u(r-R)-\left(\lambda_{0}+\lambda\right) u(r-R)\right\| \\
& \quad \leq\left\|u^{\prime \prime}(r-R)-\lambda_{0} u(r-R)\right\| \\
& \quad+\left\|\left(\frac{1}{r^{2}}\left[\frac{(\nu-1)(\nu-3)}{4}+l_{R}\left(l_{R}+\nu-2\right)\right]-\lambda\right) u(r-R)\right\| \leq \varepsilon\|\phi\|,
\end{aligned}
$$

which concludes the proof.

Once the essential spectrum is localized, we can turn to its continuous component. In view of the decomposition (2.1) we have to describe the continuous spectrum in each partial wave and the results for $\mathrm{H}_{\Lambda}$ will immediately follow; recall that the essential spectrum of $\mathrm{H}_{l}$ consists of the bands of the underlying one-dimensional operator $h_{\Lambda}$. Our strategy is to prove that the transfer matrix — defined in the appendix, Sec. ?? below — is bounded inside the bands, which implies that the spectrum remains absolutely continuous $[12,13]$. The following claim is a simple adaptation of the Lemma 2 from [1] to the singular case.

Lemma 4.4 Let $(a, b)$ be the interior of a band of the operator $\mathrm{h}_{\Lambda}$ in $L_{2}(\mathbb{R})$. Let further $K \subset(a, b)$ be a compact subinterval, $c \in \mathbb{R}$, and $x_{0}>0$. Then there is a number $C>0$ such that for every $\lambda \in K$ any solution $u$ of

$$
-u^{\prime \prime}+\frac{c}{r^{2}} u=\lambda u, \quad u \in D\left(\mathrm{~h}_{\Lambda}\right)
$$

with the normalization

$$
\left|u\left(x_{0}\right)\right|^{2}+\left|u^{\prime}\left(x_{0}\right)\right|^{2}=1
$$

satisfies in $\left(x_{0}, \infty\right)$ the inequality

$$
|u(x)|^{2}+\left|u^{\prime}(x)\right|^{2} \leq C .
$$

\footnotetext{
${ }^{2}$ For simplicity we allow ourselves the licence to write $\|f\| \equiv\|f(\cdot)\|=\|f(r)\|$ in the following formula.
} 
Proof: For a fixed $\lambda \in K$ the equation $\mathrm{h}_{\Lambda} w=\lambda w$ has two real-valued, linearly independent solutions, $u_{0}=u_{0}(\cdot, \lambda)$ and $v_{0}=v_{0}(\cdot, \lambda)$, such that $u_{0}, v_{0} \in D\left(\mathrm{~h}_{\Lambda}\right)$ and the functions $\left|u_{0}\right|,\left|u_{0}^{\prime}\right|,\left|v_{0}\right|,\left|v_{0}^{\prime}\right|$ are periodic, bounded, and continuous with respect to $\lambda$, cf. [8]. Without loss of generality we may assume that the determinant of the matrix

$$
Y=\left[\begin{array}{ll}
u_{0} & v_{0} \\
u_{0}^{\prime} & v_{0}^{\prime}
\end{array}\right]
$$

equals one; note that $u_{0}, v_{0}$ are real-valued and hence $\operatorname{det} Y$ is continuous at the singular points in view of to the Lemma 3.4. It is also nonzero, hence to any solution $u$ of (4.5) we can define the function

$$
y:=Y^{-1}\left[\begin{array}{c}
u \\
u^{\prime}
\end{array}\right]
$$

which satisfies

$$
y^{\prime}=A y \quad \text { on every interval }(n a,(n+1) a),
$$

where the the matrix $A$ is given by

$$
A:=-\frac{c}{x^{2}}\left[\begin{array}{cc}
u_{0} v_{0} & v_{0}^{2} \\
-u_{0}^{2} & -u_{0} v_{0}
\end{array}\right],
$$

being integrable away of zero. By a straightforward calculation we get

$$
y=\left[\begin{array}{c}
v_{0}^{\prime} u-v_{0} u^{\prime} \\
-u_{0}^{\prime} u+u_{0} u^{\prime}
\end{array}\right]
$$

and using Lemma 3.4 again we infer that $y$ is continuous at the singular points. Consequently,

$$
y(x)=\exp \left\{\int_{x_{0}}^{x} A(t) \mathrm{d} t\right\} y\left(x_{0}\right)
$$

is a solution of (4.8) and following [1] we arrive at the estimates

$$
\frac{1}{2}\left(|y|^{2}\right)^{\prime} \leq\left|\left(y, y^{\prime}\right)\right| \leq\|A\||y|^{2}
$$


and so

$$
|y(x)|^{2} \leq\left|y\left(x_{0}\right)\right|^{2} \exp \left\{2 \int_{x_{0}}^{x}\|A\|(t) \mathrm{d} t\right\} \leq\left|Y^{-1}\left(x_{0}\right)\right|^{2} \exp \left\{2 \int_{x_{0}}^{\infty}\|A\|(t) \mathrm{d} t\right\}
$$

for $x \geq x_{0}$ and every solution of (4.5) with the normalization (4.6). From

$$
\left[\begin{array}{c}
u(x) \\
u^{\prime}(x)
\end{array}\right]=Y(x) Y^{-1}\left(x_{0}\right)\left[\begin{array}{c}
u\left(x_{0}\right) \\
u^{\prime}\left(x_{0}\right)
\end{array}\right]+\int_{x_{0}}^{x} Y(x) A(t) y(t) \mathrm{d} t
$$

we then infer that the function $|u(\cdot)|^{2}+\left|u^{\prime}(\cdot)\right|^{2}$ is bounded in the interval $\left(x_{0}, \infty\right)$ which we set out to prove.

Now we are ready to describe the essential spectrum of $\mathrm{H}_{\Lambda}$.

Theorem 4.5 For $\mathrm{H}_{\Lambda}$ defined by (2.1) the following is true:

(i) For any gap $\left(E_{2 k-1}, E_{2 k}\right)$ in the essential spectrum of $\mathrm{h}_{\Lambda}$,

(a) $\mathrm{H}_{\Lambda}$ has no continuous spectrum in $\left(E_{2 k-1}, E_{2 k}\right)$, and

(b) the point spectrum of $\mathrm{H}_{\Lambda}$ is dense in $\left(E_{2 k-1}, E_{2 k}\right)$.

(ii) On any compact $K$ contained in the interior of a band of $\mathrm{h}_{\Lambda}$ the spectrum of $\mathrm{H}_{\Lambda}$ is purely absolutely continuous.

Proof: (i) By (4.1), none of the operators $\mathrm{H}_{l}, l=0,1,2, \ldots$ has a continuous spectrum in $\left(E_{2 k-1}, E_{2 k}\right)$, hence $\mathrm{H}_{\Lambda}$ has no continuous spectrum in this interval either. On the other hand, the entire interval $\left(E_{2 k-1}, E_{2 k}\right)$ is contained in the essential spectrum of $\mathrm{H}_{\Lambda}$; it follows that the spectrum of $\mathrm{H}_{\Lambda}$ in $\left(E_{2 k-1}, E_{2 k}\right)$ consists solely of eigenvalues which are necessarily dense in that interval.

(ii) The claim follows from the previous lemma and $[12,13]$. To make the article self-contained we prove in the appendix A a weaker result which still guarantees the absolute continuity of the spectrum in the bands in our singular case. 


\section{The discrete spectrum}

Recall that with the exception of the case $\nu=2, l=0$ the centrifugal term in the partial waves operators (2.2) is strictly positive, hence by the minimax principle there is no discrete spectrum below $E_{0}$. On the other hand, in the two-dimensional case Brown et al. noticed that regular radially periodic potentials give rise to bound states [3] which they named in a nationalist spirit. Subsequently Schmidt [4] proved that there are infinitely many such eigenvalues of the operator $\mathrm{H}_{0}$ below $\inf \sigma_{\text {ess }}\left(\mathrm{H}_{\Lambda}\right)$. Our aim is to show that this result persists for singular sphere interactions considered here.

Theorem 5.1 Let $\nu=2$, then except of the free case the operator $\mathrm{H}_{\Lambda}$ has infinitely many eigenvalues in $\left(-\infty, E_{0}\right)$, where $E_{0}:=\inf \sigma_{\mathrm{ess}}\left(\mathrm{H}_{\Lambda}\right)$.

Proof: The argument is again similar to that of the regular case [4], hence we limit ourselves to just sketching it. First of all, it is clear that we have to investigate the spectrum of $\mathrm{H}_{\Lambda, 0}$.

Let $u, v$ be linearly independent real-valued solutions of the equation $\mathrm{h}_{\Lambda} z=E_{0} z$, where $u$ is (anti)periodic - cf. Proposition 3.1. - satisfying $W[u, v]=1$. We will search the solution of $\mathrm{H}_{0} y \equiv-y^{\prime \prime}-\frac{1}{4 r^{2}} y=E_{0} y$, we are interested in, using a Prüfer-type Ansatz, namely

$$
\left(\begin{array}{c}
y \\
y^{\prime}
\end{array}\right)=\left(\begin{array}{cc}
u & v \\
u^{\prime} & v^{\prime}
\end{array}\right) a\left(\begin{array}{c}
\sin \gamma \\
-\cos \gamma
\end{array}\right)
$$

where $a$ is a positive function and $\gamma$ is chosen continuous recalling Lemma 3.4 and eq. (3.4). It is demonstrated in [4] that the function $\gamma(\cdot)$ and the standard Prüfer variable $\theta(\cdot)$, appearing in

$$
\left(\begin{array}{c}
y \\
y^{\prime}
\end{array}\right)=\rho\left(\begin{array}{c}
\cos \theta \\
\sin \theta
\end{array}\right),
$$

are up to constant asymptotically equal to each other as $r \rightarrow \infty$. According to Corollary B.3 there are then infinitely many eigenvalues below $E_{0}$ if $\theta$, and therefore also $\gamma$, is unbounded from below.

Now a straightforward computation yields

$$
\gamma^{\prime}=-\frac{1}{4 r^{2}}(u \sin \gamma-v \cos \gamma)^{2}=-\frac{1}{4} \cos ^{2} \gamma u^{2}\left(\frac{1}{r} \tan \gamma-\frac{v}{r u}\right)^{2}
$$


Furthermore, the Kepler transformation given by the relation $\tan \phi=\left(r^{-1} \tan \gamma-\right.$ $\left.r^{-1} v / u\right)$ satisfies $\gamma(r)=\phi(r)+\mathcal{O}(1)$ as $r \rightarrow \infty$, and

$$
\begin{aligned}
\phi^{\prime}=\frac{1}{r}(-\sin \phi \cos \phi & \left.-\frac{1}{4} u^{2} \sin ^{2} \phi-\frac{1}{u^{2}} \cos ^{2} \phi\right) \\
& =-\frac{1}{2 r}\left(\frac{1}{u^{2}}+\frac{1}{4} u^{2}+\sin 2 \phi+\left(\frac{1}{u^{2}}-\frac{1}{4} u^{2}\right) \cos 2 \phi\right)
\end{aligned}
$$

holds on $\mathbb{R} \backslash \cup_{n}\left\{r_{n}\right\}$ with the discontinuity

$$
\tan \phi\left(r_{n}+\right)-\tan \phi\left(r_{n}-\right)=-\frac{1}{r_{n}} \frac{\beta}{u\left(r_{n}+\right) u\left(r_{n}-\right)},
$$

where $\beta$ is the parameter appearing in (2.3). A direct analysis of the equation (5.1) shows that $\phi^{\prime} \leq 0$, and owing to (5.2) and Proposition 3.1 the corresponding discontinuity is strictly negative for $\beta \neq 0$. Hence $\phi$ is decreasing and there is a limit $L=\lim _{r \rightarrow \infty} \phi(r)$. Suppose that $L$ is finite. Then the condition $\left|\int_{0}^{\infty} \phi^{\prime}(t) \mathrm{d} t\right|<\infty$ gives

$\frac{1}{u^{2}(r)}+\frac{1}{4} u^{2}(r)+\sin 2 \phi(r)+\left(\frac{1}{u^{2}(r)}-\frac{1}{4} u^{2}(r)\right) \cos 2 \phi(r) \rightarrow 0 \quad$ as $\quad r \rightarrow \infty$

and, as $u$ is (anti)-periodic and $\phi$ tends to a constant, we infer that $u^{2}$ is constant also, not only asymptotically but everywhere. With the exception of the free case this may happen only for pure repulsive $\delta^{\prime}$ interaction, $\beta>0, \alpha=0, \gamma=\delta=1$. To finish the proof we employ eq. (5.3) again and observe that $L \neq \pi / 2(\bmod \pi)$ holds necessarily. We thus find a monotonous sequence of points $r_{n}$ such that $\phi\left(r_{n}-\right)<\frac{\pi}{2}\left(1+\left[\frac{2 L}{\pi}\right]\right)$, where [.] is the integer part. Since $\phi$ is monotonous we have $\phi\left(r_{n} \pm\right) \geq L$, hence all these points belong to the same branch of the tan function. Summing then the discontinuities (5.2) we get

$$
\begin{aligned}
\tan \phi\left(r_{N}+\right)-\tan \phi\left(r_{n}-\right) & \leq \sum_{i=n}^{N} \tan \phi\left(r_{i}+\right)-\tan \phi\left(r_{i}-\right) \\
& =-\sum_{i=n}^{N} \frac{1}{r_{i}} \frac{\beta}{u\left(r_{i}+\right) u\left(r_{i}-\right)},
\end{aligned}
$$

where the right-hand side diverges as $N \rightarrow \infty$ for any $\beta>0$, while the left-hand side tends to a finite number $\tan (L)-\tan \phi\left(r_{n}-\right)$. Hence $L$ can be finite for the free Hamiltonian only, which was to be demonstrated. 


\section{A Continuous spectra for one dimensional Schrödinger operators with singular inter- actions}

In this appendix we consider Schrödinger operators on a halfline,

$$
\begin{aligned}
& (\mathrm{H} u)(x)=-u^{\prime \prime}(x)+V(x) u(x) \\
& u(0)=0, \quad U\left(x_{n}+\right)=\Lambda U\left(x_{n}-\right),
\end{aligned}
$$

where we suppose that the condition

$$
\int_{K}^{\infty}\left|u^{\prime}\right|^{2} \leq \beta \int_{K}^{\infty}\left(|\mathrm{H} u|^{2}+|u|^{2}\right)
$$

holds for some $\beta, K>0$ and every $u \in D(\mathrm{H})$. This is obviously the case of operators $\mathrm{H}_{\lambda, l}$, where in the dimension $\nu>2$ we may put $K=0$, while for $\nu=2$ we have to choose $K>0$.

Given a solution $u$ of $\mathrm{H} u=E u$ we define the transfer matrix $\mathrm{T}(E, x, y)$ at energy $E$ by

$$
\mathrm{T}(E, x, y)\left(\begin{array}{l}
u^{\prime}(y) \\
u(y)
\end{array}\right)=\left(\begin{array}{l}
u^{\prime}(x) \\
u(x)
\end{array}\right) .
$$

Our purpose is to prove the following result.

Theorem A.1 Let $\mathrm{T}(E, x, y)$ be bounded on $S$. Then for every interval $\left(E_{1}, E_{2}\right) \subset S$ we have $\rho_{a c}\left(\left(E_{1}, E_{2}\right)\right)>0$ and $\rho_{s c}\left(\left(E_{1}, E_{2}\right)\right)=0$, where $\rho$ denotes the spectral measure associated with the operator $\mathrm{H}$.

Following [13] we employ the theory of Weyl m-functions. For $E \in C_{+}=$ $\{z, \operatorname{Im} z>0\}$, there is a unique solution $u_{+}(x, E)$ of $\mathrm{H} u_{+}(x, E)=E u_{+}(x, E)$ with $u_{+} \in L^{2}$ at infinity, which is normalized by $u_{+}(0, E)=1$. We define the m-function by

$$
m_{+}(E)=u_{+}^{\prime}(0, E) ;
$$

the spectral measure $\rho$ is then related to it by

$$
\mathrm{d} \rho(E)=\frac{1}{\pi} \lim _{\varepsilon \downarrow 0} \operatorname{Im} m_{+}(E+i \varepsilon),
$$

where the imaginary part at the right-hand side can be expressed as

$$
\operatorname{Im} m_{+}(E)=\operatorname{Im} E \int_{0}^{\infty}\left|u_{+}(x, E)\right|^{2} \mathrm{~d} x .
$$


It is known, see [13] and references therein, that

$$
\operatorname{supp} \rho_{s c}=\left\{E: \lim _{\varepsilon \downarrow 0} \operatorname{Im} m_{+}(E+i \varepsilon)=\infty\right\}
$$

while $\mathrm{d} \rho_{a c}(E)=\frac{1}{\pi} \operatorname{Im} m_{+}(E+i 0) \mathrm{d} E$. Theorem A.1 is then an immediate consequence of the following result.

Theorem A.2 If $\mathrm{T}(E, x, y)$ be bounded as above and $E \in\left(E_{1}, E_{2}\right)$, then

$$
\liminf \operatorname{Im} m_{+}(E+i 0)>0 \text { and } \limsup \operatorname{Im} m_{+}(E+i 0)<\infty
$$

Proof: For $x \neq x_{n}$ we have the relations

$$
\begin{aligned}
& \frac{\mathrm{d} \mathrm{T}(E, x, y)}{\mathrm{d} x}=\left(\begin{array}{cc}
0 & V(x)-E \\
1 & 0
\end{array}\right) \mathrm{T}(E, x, y), \\
& \frac{\mathrm{d}}{\mathrm{d} y}\left(\left(\mathrm{~T}\left(E_{1}, x, y\right) \mathrm{T}\left(E_{2}, y, x\right)\right)=\left(E_{1}-E_{2}\right) \mathrm{T}\left(E_{1}, x, y\right)\left(\begin{array}{ll}
0 & 1 \\
0 & 0
\end{array}\right) \mathrm{T}\left(E_{2}, y, x\right) .\right.
\end{aligned}
$$

It is straightforward to verify that $\mathrm{T}\left(E_{1}, x, y\right) \mathrm{T}\left(E_{2}, y, x\right)$ is continuous at singular points with respect to $y$ and hence

$$
1-\mathrm{T}\left(E_{1}, x, 0\right) \mathrm{T}\left(E_{2}, 0, x\right)=\int_{0}^{x}\left(E_{1}-E_{2}\right) \mathrm{T}\left(E_{1}, x, y\right)\left(\begin{array}{ll}
0 & 1 \\
0 & 0
\end{array}\right) \mathrm{T}\left(E_{2}, y, x\right) \mathrm{d} y \text {. }
$$

Now we put $E_{1}=E, E_{2}=E+i \varepsilon$ and multiply by $\mathrm{T}(E+i \varepsilon, x, 0)$ from the right to get the formula

$$
\mathrm{T}(E+i \varepsilon, x, 0)=\mathrm{T}(E, x, 0)-(i \varepsilon) \int_{0}^{x} \mathrm{~T}(E, x, y)\left(\begin{array}{ll}
0 & 1 \\
0 & 0
\end{array}\right) \mathrm{T}(E+i \varepsilon, y, 0) \mathrm{d} y .
$$

By assumption we have $\|\mathrm{T}(E, x, y)\| \leq C$, and therefore

$$
\|\mathrm{T}(E+i \varepsilon, x, 0)\| \leq C+\varepsilon \int_{0}^{x} C\|\mathrm{~T}(E+i \varepsilon, y, 0)\| \mathrm{d} y
$$

so by iteration we get

$$
\|\mathrm{T}(E+i \varepsilon, x, 0)\| \leq C e^{\varepsilon C x} .
$$


Note that $\operatorname{det} \mathrm{T}=1$ so $\|\mathrm{T}\|=\left\|\mathrm{T}^{-1}\right\|$. Putting now $\gamma=\left((E+1)^{2} \beta^{2}+1\right)^{-1}$ and using the condition (1.3) we get

$$
\begin{aligned}
\int_{0}^{\infty}|u(x)|^{2} \mathrm{~d} x & \geq \gamma \int_{K}^{\infty}\left(|u(x)|^{2}+\left|u^{\prime}(x)\right|^{2}\right) \mathrm{d} x \\
& \geq C^{-2} \gamma\left(1+\left|m_{+}\right|^{2}\right) \int_{K}^{\infty} e^{-2 \varepsilon C x} \mathrm{~d} x
\end{aligned}
$$

hence by (1.5) we infer that

$$
\operatorname{Im} m_{+} \geq \frac{1}{2} C^{-3} \gamma\left(1+\left|m_{+}\right|^{2}\right) .
$$

From here the first claim follows immediately, and since

$$
2 C^{3} \gamma^{-1} \geq \frac{1+\left|m_{+}\right|^{2}}{\operatorname{Im} m_{+}} \geq\left|m_{+}\right|
$$

we get also the remaining part.

\section{B Oscillation theory for singular potentials}

In the case of point interactions the classical oscillation theory fails due to discontinuity of the wave functions. Nevertheless, we can employ the continuity of the Wronskian and formulate the oscillation theory using the approach of relative oscillations [14]. The aim of this appendix is to present briefly the basic theorems; since the claims are the same as in the regular case we follow closely the above mentioned article.

We consider Schrödinger-type operators on $L^{2}\left(l_{-}, l_{+}\right)$with the singular interactions at the points $x_{n} \in\left(l_{-}, l_{+}\right), n \in M \subset \mathbb{N}$ which act as

$$
\mathrm{T} u(x)=-u^{\prime \prime}(x)+q(x) u(x),
$$

with a real-valued potential $q \in L_{\mathrm{loc}}^{1}\left(l_{-}, l_{+}\right)$and the domain

$$
\begin{aligned}
& D(\mathrm{~T})=\left\{u, u^{\prime} \in A C_{\mathrm{loc}}\left(l_{-}, l_{+}\right) \backslash \bigcup_{n \in M}\left\{x_{n}\right\}:\right. \\
& \left.T u \in L_{\mathrm{loc}}^{2}\left(l_{-}, l_{+}\right) \text {and } U\left(x_{n}+\right)=\Lambda_{n} U\left(x_{n}-\right)\right\} .
\end{aligned}
$$


Such an operator is obviously symmetric. Denote by $\mathrm{H}$ an arbitrary selfadjoint extension of it satisfying either

(a) $\mathrm{T}$ is limit point in at least one endpoint, or

(b) $\mathrm{H}$ is defined by separated boundary conditions.

By $\psi_{ \pm}(E, x)$ we denote real-valued solutions of the equation $\mathrm{T} \psi_{ \pm}(E, x)=$ $E \psi_{ \pm}(E, x)$, which satisfy the boundary conditions defining $\mathrm{H}$ at the points $l_{ \pm}$, respectively. Note that such solutions may not exist, the theorems given below implicitly assume their existence. In particulary, their existence is assure for energies $E$ outside the essential spectrum. And with respect to analyticity in spectral parameter we may use the oscillation theory also at the edge of the essential spectrum.

The first theorem to follow provides the basic oscillation result, while the corollary of the second one is the result used in Section 5. By $W_{0}\left(u_{1}, u_{2}\right)$ we denote the number of zeros of the Wronskian $W\left[u_{1}, u_{2}\right](x)$ in the open interval $\left(l_{-}, l_{+}\right)$, and given $E_{1}<E_{2}$, we put $N_{0}\left(E_{1}, E_{2}\right)=\operatorname{dim} \operatorname{RanP}_{\left(E_{1}, E_{2}\right)}$, where $\mathrm{P}$ is a spectral measure of the self-adjoint operator $\mathrm{H}$. In particular, in case of the pure point spectrum $N_{0}\left(E_{1}, E_{2}\right)$ simply denotes the number of eigenvalues in the interval $\left(E_{1}, E_{2}\right)$.

Theorem B.1 Suppose that $E_{1}<E_{2}$ and put $u_{1}=\psi_{-}\left(E_{1}\right), u_{2}=\psi_{+}\left(E_{2}\right)$. Then $W_{0}\left(u_{1}, u_{2}\right)=N_{0}\left(E_{1}, E_{2}\right)$.

Theorem B.2 Let $E_{1}<E_{2}$. Assume that either $u_{1}=\psi_{+}\left(E_{1}\right)$ or $u_{1}=$ $\psi_{-}\left(E_{1}\right)$ holds, and similarly either $u_{2}=\psi_{+}\left(E_{2}\right)$ or $u_{2}=\psi_{-}\left(E_{2}\right)$. Then $W_{0}\left(u_{1}, u_{2}\right) \leq N_{0}\left(E_{1}, E_{2}\right)$.

Next we introduce Prüfer variables $\rho_{i}, \theta_{i}$ defined by

$$
\left(\begin{array}{c}
u_{i}(x) \\
u_{i}^{\prime}(x)
\end{array}\right)=\rho_{i}(x)\left(\begin{array}{c}
\cos \theta_{i}(x) \\
\sin \theta_{i}(x)
\end{array}\right)
$$

where $\rho_{i}$ is chosen positive and $\theta_{i}$ is uniquely determined by its boundary value and the requirement that $\theta_{i}$ is continuous on $\left(l_{-}, l_{+}\right) \backslash \bigcup_{n \in M}\left\{x_{n}\right\}$ while its discontinuity at the sites $x_{n}$ of the point interactions satisfies $\mid \theta_{i}\left(x_{n}+\right)-$ $\theta_{i}\left(x_{n}-\right) \mid=0(\bmod \pi)$.

Corollary B.3 Suppose that $E_{1}$ is the edge of the essential spectrum, and $u_{1}=\psi_{-}\left(E_{1}\right)$ or $u_{1}=\psi_{+}\left(E_{1}\right)$. Then $\mathrm{H}$ has infinitely many eigenvalues below $E_{1}$ if $\theta_{1}(\cdot)$ is unbounded. 
Proof: In analogy with the regular case the function $\theta_{2}$ corresponding to $u_{2}=\psi_{ \pm}(E)$ is bounded for negative $E$ large enough. This implies that $\left|\theta_{2}-\theta_{1}\right| \rightarrow \infty$ and since $W\left[u_{1}, u_{2}\right](x)=\rho_{1}(x) \rho_{2}(x) \sin \left(\theta_{2}(x)-\theta_{1}(x)\right)$ we get $W_{0}\left(u_{1}, u_{2}\right)=\infty$. Hence Theorem B.2. completes the proof.

\section{Acknowledgment}

The research was supported by the Czech Ministry of Education, Youth and Sports within the project LC06002.

\section{References}

[1] R. Hempel, I. Herbst, A.M. Hinz, H. Kalf, J. London Math. Soc. 43, 295-304 (1991).

[2] P. Exner, M. Fraas, Lett. Math. Phys. 82, 25-37 (2007).

[3] B.M. Brown et al., J. Math. Anal. Appl. 225, 347-357 (1998).

[4] K.M. Schmidt, Commun. Math. Phys. 211, 465-485 (2000).

[5] P. Exner, M. Fraas, Phys. Lett. A360, 57-61 (2006).

[6] T. Cheon, T. Shigehara, J. Phys. Soc. Japan 73, 2986-2990 (2004).

[7] P. Exner, H. Grosse, mp_arc 99-390 or math-ph/9910029.

[8] J. Weidmann, Spectral Theory of Ordinary Differential Operators (Springer, Berlin, 1987).

[9] R.M. Redheffer, Math. Zeitschr. 80, 390-397 (1963).

[10] R. Hempel, A.M. Hinz, H. Kalf, Math. Ann. 277, 197-208 (1987).

[11] J. Weidmann, Math. Ann. 277, 209-211 (1987).

[12] D.J. Gilbert, D.B. Pearson, J. Math. Anal. Appl. 128, 30-56 (1987).

[13] B. Simon, Proc. Amer. Math. Soc. 124, 3361-3369 (1996).

[14] F. Gesztesy, B. Simon, G. Teschl, Am. J. Math. 118 571-594, (1996). 